\title{
Controlling Synchrony in a Network of Kuramoto Oscillators with Time-Varying Coupling
}

\author{
Rachel Leander ${ }^{\mathrm{a}, *}$, Suzanne Lenhart ${ }^{\mathrm{b}}$, Vladimir Protopopescu ${ }^{\mathrm{c}}$ \\ ${ }^{a}$ Department of Mathematical Sciences, Middle Tennessee State University, \\ Murfreesboro, TN 37132 \\ ${ }^{b}$ Mathematics Department, University of Tennessee, Knoxville, TN 37996 \\ ${ }^{c}$ Computational Sciences and Engineering Division, Oak Ridge National Laboratory, Oak \\ Ridge, TN 37831
}

\begin{abstract}
The Kuramoto model describes the synchronization of a heterogeneous population of oscillators through a stationary homogeneous network in which oscillators are coupled via their phase differences. Recently, there has been interest in studying synchronization on time-varying networks, and timevarying generalizations of the Kuramoto network, in particular. Previous results indicate that networks with fast dynamics may be as efficient as static networks at promoting synchrony. In this paper we use optimal control theory to study synchronization on a time-varying Kuramoto network. Our results indicate that time-varying networks can be more efficient than static networks at promoting synchrony and show that fast network dynamics are not necessary for efficiency. In particular, we show that, near the synchronization threshold, time-varying networks can promote synchrony through slow oscillations that lengthen the duration of high synchrony states and shorten the duration of low synchrony states. Interestingly, repulsion is an essential feature of these optimal dynamic networks.
\end{abstract}

Keywords: synchrony, time-varying coupling, Kuramoto oscillators

\footnotetext{
*corresponding author

Email addresses: rachel.leander@mtsu.edu (Rachel Leander), lenhart@utk.edu (Suzanne Lenhart), protopopesva@ornl.gov (Vladimir Protopopescu)
} 


\section{Introduction}

Individuals within a population may synchronize their dynamics if they are able to interact via a network. The structure of the network and the form of the interaction that takes place on the network are important determinants of synchrony. Several authors have considered the relation between network structure and synchrony, under the assumption that the network is static $[1,2,3]$. More recently, there has been interest in studying synchrony on networks that change through time. Several papers have examined synchrony on networks in which the weights of the links/edges are dynamic $[4,5,6,7]$. Such networks can be used to model intermittent communication, as is the case for blinking networks in which the edges switch on and off according to some probability distribution [4], or more general networks that can switch between multiple states [7]. In [7], it was shown that, if the switching is fast enough, a population interacting over a switching network can achieve the same level of synchrony as a population interacting over a static network with the same time-averaged connectivity. Similarly, the blinking network considered in [4] was shown to support synchrony if the blinking is fast enough, and the threshold for synchronization was related to that of a corresponding averaged network. In addition, under certain conditions, it was shown that a blinking system may converge asymptotically to an attractor of the corresponding time-averaged system [5]. More recently still, there has been interest in evolving networks, i.e. in networks that change in response to the state of their nodes (see [8] for a recent review). Networks that adapt in order to promote synchronization are of particular interest $[7,9,10]$. In these works, the network dynamics are determined by a specific rule (e.g. edges are turned on and off with a given probability, the weight of an edge grows as the disparity between its vertices increases), and the dynamics of the resulting system are examined.

In this paper, we use optimal control theory in order to construct optimal time-varying networks. In particular, our work differs from previous research in that the network dynamics are identified using the necessary conditions associated with an optimal control problem. These networks are optimal in that they minimize/maximize objective functionals that depend on the synchrony of the population and the connectivity of the network, under the assumption that synchrony is beneficial and connectivity is costly.

In [3] we used optimal control theory to examine the properties of opti- 
mal static networks and, in particular, to determine how the heterogeneity of an optimal static network depends on the underlying heterogeneity of the population that the network joins. Due to the complexity of the optimal timevarying networks, this work focuses on characterizing optimal time-varying couplings between small groups of oscillators, beginning with a single oscillator pair. In the future, insights into the principles that govern optimal time-varying couplings between a small group of oscillators can be used to interpret results for large populations and identify size-dependent features of optimal time-dependent couplings. Here, we show that close to the synchronization threshold, dynamic networks are more efficient than static networks, be they heterogeneous or homogeneous, at promoting synchrony. In addition, numerical simulations indicate that dynamic networks use repulsion to enhance network synchrony, in one instance, even driving a contrarian oscillator to have negative velocity (See Figure $6(\mathrm{~b})$ ), and lead to large amplitude oscillations of the network's order parameter.

Our analysis is based on the Kuramoto model, which is an elegant mathematical model of nonlinear phase oscillators that is capable of producing synchrony. The Kuramoto model is a system of nonlinear, coupled, ordinary differential equations [11]:

$$
\frac{d x_{i}}{d t}=\omega_{i}+\frac{K}{N} \sum_{j=1}^{N} \sin \left(x_{j}(t)-x_{i}(t)\right),
$$

where $N$ is the number of oscillators in the network, $x_{i}$ is the phase of the $i^{t h}$ oscillator, and $\omega_{i}$ is the natural velocity of the $i^{t h}$ oscillator. The coefficient $K$ is called the coupling strength. The complex valued order function,

$$
r(t) \mathrm{e}^{i \psi(t)}=\frac{1}{N} \sum_{j=1}^{N} \mathrm{e}^{i x_{j}(t)},
$$

quantifies the network's state. Here $\psi$ denotes the centroid of the oscillator phases, and $r(t)$ provides a quantitative measure of network synchrony. In particular, $r(t)=1$ corresponds to perfect synchrony, and $r(t)=0$ corresponds to a state in which the oscillators' phases are evenly distributed around the unit circle [11]. We will refer to $r(t)$ as the order parameter of the system.

The state dynamics of the Kuramoto model can be rewritten in terms of 
the order parameter as follows:

$$
\frac{d x_{i}}{d t}=\omega_{i}+r(t) K \sin \left(\psi(t)-x_{i}(t)\right),
$$

so that each oscillator is coupled to the mean phase with strength $K r(t)$ $[11,12]$. The network's dynamics depend on $K$ as follows [12]. If $K$ is small, the order parameter of the Kuramoto network fluctuates. There is a critical coupling threshold above which the oscillators become phase-locked and the order parameter stabilizes to a constant. As the coupling strength increases further, the value of the order parameter grows too. A more detailed account of the Kuramoto model and some bounds on the value of the critical coupling strength can be found in [12].

In the Kuramoto network model, the coupling strength is constant and is independent of time. In this paper, we consider a generalization of the Kuramoto model in which the coupling strength may vary between oscillator pairs and also through time. In particular, we consider the following Kuramoto type model with time varying-coupling:

$$
\frac{d x_{i}}{d t}=\omega_{i}+\frac{1}{N} \sum_{j=1}^{N} u_{i j}(t) \sin \left(x_{j}(t)-x_{i}(t)\right) .
$$

Assuming that network synchrony is desirable and that there is a cost associated with network connectivity, we use optimal control theory to design networks that can efficiently promote synchrony among a set of oscillators. The set of oscillators will be characterized by two vectors, $x_{0}$ and $\omega$, that represent the oscillators' initial phases and natural velocities, respectively. Given $x_{0}$ and $\omega$, we will use optimal control theory to find the coupling strengths, $u_{i j}$, that maximize an appropriate objective functional. In particular, the coupling strengths will serve as control variables, and their magnitudes as a measure of network connectivity. Note that although, in principle, the matrix of coupling strengths could have nonzero diagonal elements, the form of the state dynamics implies that the oscillators cannot influence their own dynamics. Hence, in an optimal network, for $i=1, \ldots, N, u_{i i}^{*}=0$. For this reason, when computing the cost of the Kuramoto network, we will also treat the diagonal elements as zero. For the purpose of formulating an objective functional, the network synchrony and the network connectivity cost are quantified as follows. The network synchrony is defined to be

$$
\int_{0}^{T} r^{2}(t) d t
$$


and the connectivity cost over the interval $[0, T]$ is defined to be

$$
\int_{0}^{T} \sum_{i j} u_{i j}(t)^{2} d t .
$$

The quadratic form of the coupling cost is important for identifying the optimal control and interpreting results. In particular, although intuitively the total connectivity of the network should determine the level of population synchrony, it is easily seen that for fixed total connectivity, i.e. for $\int_{0}^{T} \sum_{i j}\left|u_{i j}(t)\right| d t$ fixed, there can be multiple networks that achieve the same level of synchrony. The quadratic form of the connectivity cost, however, implies that among networks with the same total connectivity, those in which the connectivity is distributed evenly through time and among network edges have lower connectivity cost; i.e. the quadratic form of the connectivity cost penalizes dynamic and heterogeneous networks. Hence, temporal dynamics and network heterogeneity that are present in an optimal network must function to enhance the population's synchrony.

When comparing solutions, it is also useful to consider the average network synchrony and average connectivity cost:

$$
\begin{gathered}
\frac{1}{T} \int_{0}^{T} r^{2}(t) d t \\
\frac{1}{T N(N-1)} \int_{0}^{T} \sum_{i j} u_{i j}(t)^{2} d t .
\end{gathered}
$$

Note that since the oscillators in a Kuramoto-type network cannot influence their own dynamics we have normalized the connectivity cost by $\frac{1}{N(N-1)}$, to reflect the fact that the system has, in effect, only $N(N-1)$ controls. For the same reason, when computing the connectivity cost for a standard Kuramoto network, we treat the diagonal elements $u_{i i}=0$. With these definitions in place, we address the following three questions.

(1) What is the least connected network capable of obtaining a prescribed level of synchrony?

(2) How can we achieve the greatest level of synchrony in a network with bounded connectivity?

(3) How can we optimize synchrony vs. connectivity cost? 
We begin each section by formulating the relevant optimal control problem. After the problem is formulated, we proceed to show the existence of optimal coupling strengths and to characterize the optimal coupling strengths with the aid of Pontryagin's maximum principle. Characterization of the optimal coupling strengths is nontrivial for the first two problems, due to the integral constraints imposed on the controls and the states. Thus, we provide detailed proofs that the characterizations are correct. Once the optimal coupling strengths are characterized, we perform numerical simulations. Each numerical simulation answers one of the above questions for a specific network. In the final section of this article, we summarize our observations and suggest future directions of research.

\section{Minimizing the Connectivity Cost of a Network with Prescribed Synchrony}

In this section, given the initial phases and natural velocities of a set of oscillators $\left(x_{0}\right.$ and $\left.\omega\right)$, we find the coupling strengths $u_{i j}(t)$ that enable the set of oscillators to achieve a prescribed level of synchrony at the minimal possible connectivity cost. Later we will compare the efficacy of the timevarying network to that of the classical Kuramoto network. To this end, we set the prescribed level of synchrony equal to that of a Kuramoto network with coupling strength $K$. Specifically, given $x_{0}, \omega$, and $K$ we will find the coupling strengths, $u_{i j}^{*}(t)$, that minimize

$$
J_{1}(u):=\int_{0}^{T} \sum_{i j} u_{i j}^{2}(t) d t,
$$

subject to

$$
\int_{0}^{T} r^{2}(t) d t=A
$$

where $A$ is the synchrony obtained by an identical set of oscillators interacting through the Kuramoto network with $u_{i j}(t) \equiv K$. The control set $U$ is defined as $U=[-M, M]^{N \times N}$, i.e. the set of all $N \times N$ matrices with elements in $[-M, M]$. Controls for this problem are functions $u:[0, T] \rightarrow U$, such that each component $u_{i j}(t)$ is a Lebesgue measurable function from $[0, T]$ to $[-M, M]$. To incorporate the constraint $\int_{0}^{T} r^{2}(t) d t=A$ into the problem, we 
introduce an artificial state variable $x_{N+1}$, which satisfies:

$$
\begin{aligned}
\frac{d x_{N+1}}{d t} & =r^{2}(t) \\
& =\frac{1}{N^{2}}\left[\left(\sum_{i=1}^{N} \sin x_{i}(t)\right)^{2}+\left(\sum_{i=1}^{N} \cos x_{i}(t)\right)^{2}\right] \\
& =\frac{1}{N^{2}} \sum_{i, j=1}^{N} \sin x_{i}(t) \sin x_{j}(t)+\cos x_{i}(t) \cos x_{j}(t) \\
& =\frac{1}{N^{2}} \sum_{i, j=1}^{N} \cos \left(x_{i}(t)-x_{j}(t)\right)
\end{aligned}
$$

and

$$
x_{N+1}(0)=0, \quad x_{N+1}(T)=A .
$$

We say that a vector of state trajectories $x(t)=\left(x_{1}(t), \ldots, x_{N}(t), x_{N+1}(t)\right)$ corresponds to the control $u(t)$ if the components of $x(t)$ are absolutely continuous functions which satisfy

$\frac{d x_{i}}{d t}=\omega_{i}+\frac{1}{N} \sum_{j=1}^{N} u_{i j}(t) \sin \left(x_{j}(t)-x_{i}(t)\right) ; \quad x_{i}(0)=x_{i}^{0} \quad$ for $i \in\{1,2, \ldots, N\}$

and

$$
\frac{d x_{N+1}}{d t}=\frac{1}{N^{2}} \sum_{i, j=1}^{N} \cos \left(x_{i}(t)-x_{j}(t)\right) ; \quad x_{N+1}(0)=0 .
$$

In this case, we call $(x(t), u(t))$ a trajectory-control pair.

The set of admissible controls, $U_{a d}$, is defined as follows: Let $(x(t), u(t))$ be a trajectory-control pair, with $u:[0, T] \rightarrow U$. We say that $u \in U_{a d}$ if $x_{N+1}(T)=A$. Note that $U_{a d} \neq \emptyset$, because the control with $u_{i j}(t) \equiv K$ is an element of $U_{a d}$ by design.

Within this framework, the problem may be stated as follows:

Problem I:

Find a trajectory-control pair $\left(x^{*}, u^{*}\right)$ so that $u^{*} \in U_{a d}$, and

$$
J_{1}\left(u^{*}\right)=\min _{u \in U_{a d}} J_{1}(u)
$$


Theorem 2.1. There exists a solution to Problem I.

Proof. Since $J_{1}(u)$ is bounded on $U_{a d}$, we can choose a sequence $\left\{u^{m}\right\} \in U_{a d}$ so that $J_{1}\left(u^{m}\right) \rightarrow \inf _{u \in U_{a d}} J_{1}(u)$. Since the control components, $u_{i j}^{m}$, are $L^{2}[0, T]$ bounded, there exist $L^{2}$ functions, $u_{i j}^{*}$, and a subsequence (which we will also denote by $\left\{u^{m}\right\}$ ), so that $u_{j k}^{m}$ converges weakly to $u_{i j}^{*}$ in $L^{2}[0, T]$. If $x^{m}$ is the state associated with $u^{m}$, we have

$$
\frac{d x_{j}^{m}}{d t}=\omega_{j}+\frac{1}{N} \sum_{k=1}^{N} u_{j k}^{m}(t) \sin \left(x_{k}^{m}(t)-x_{j}^{m}(t)\right), \quad j \in\{1, \ldots, N\},
$$

and so, for $t_{2}>t_{1}$,

$$
\begin{aligned}
\left|x_{j}^{m}\left(t_{2}\right)-x_{j}^{m}\left(t_{1}\right)\right| & \leq\left|\int_{t_{1}}^{t_{2}} \omega_{j}+\frac{1}{N} \sum_{k=1}^{N} u_{j k}^{m}(t) \sin \left(x_{k}^{m}(t)-x_{j}^{m}(t)\right) d t\right| \\
& \leq\left(t_{2}-t_{1}\right) \max _{i}\left\{\omega_{i}\right\}+\frac{1}{N} \int_{t_{1}}^{t_{2}} \sum_{i}\left|u_{i j}^{m}(t)\right| d t \\
& \leq\left(t_{2}-t_{1}\right) \max _{i}\left\{\omega_{i}\right\}+\left(t_{2}-t_{1}\right)(M) .
\end{aligned}
$$

Thus, for $W=\max _{i \in\{1, \ldots, N\}}\left(\omega_{i}, M\right)$ and $\left(t_{2}-t_{1}\right) \leq \frac{\epsilon}{W},\left|x_{j}^{m}\left(t_{2}\right)-x_{j}^{m}\left(t_{1}\right)\right| \leq \epsilon$. Thus, the functions $\left\{x_{j}^{m}\right\}$ are equicontinuous. Furthermore, since

$$
\begin{gathered}
\left|x_{j}^{m}(s)\right|=\left|x_{j}^{0}+\int_{0}^{s} \omega_{j}+\frac{1}{N} \sum_{k=1}^{N} u_{j k}^{m}(t) \sin \left(x_{k}^{m}(t)-x_{j}^{m}(t)\right) d t\right| \\
\leq \max _{i}\left\{x_{i}^{0}\right\}+T \max _{i}\left\{\omega_{i}\right\}+T M,
\end{gathered}
$$

the functions $\left\{x_{j}^{m}\right\}$ are uniformly bounded. Since the functions $\left\{x_{N+1}^{m}\right\}$ are trivially equicontinuous and uniformly bounded, we conclude that there exists a subsequence, $\left\{x_{j}^{m}\right\}$, that converges uniformly to $x_{j}^{*}$ in $C[0, T]$. In particular, it follows that

$$
x_{N+1}^{*}(T)=\lim _{m \rightarrow \infty} x_{N+1}^{m}(T)=A .
$$

Next we show that

$$
\frac{d x_{j}^{*}}{d t}=\omega_{j}+\frac{1}{N} \sum_{k=1}^{N} u_{j k}^{*}(t) \sin \left(x_{k}^{*}(t)-x_{j}^{*}(t)\right)
$$


in $H^{1}[0, T]$. To see this, note that for $v \in C^{\infty}[0, T]$

$$
\begin{aligned}
& \lim _{m \rightarrow \infty} \int_{0}^{T} v(t) \sum_{k} u_{j, k}^{*}(t) \sin \left(x_{k}^{*}(t)-x_{j}^{*}(t)\right)-u_{j k}^{m}(t) \sin \left(x_{k}^{m}(t)-x_{j}^{m}(t)\right) d t \\
= & \lim _{m \rightarrow \infty} \int_{0}^{T} v(t) \sum_{k}\left(u_{j, k}^{*}(t)-u_{j k}^{m}(t)\right) \sin \left(x_{k}^{*}(t)-x_{j}^{*}(t)\right) d t \\
+ & \lim _{m \rightarrow \infty} \int_{0}^{T} v(t) \sum_{k}\left[\sin \left(x_{k}^{*}(t)-x_{j}^{*}(t)\right)-\sin \left(x_{k}^{m}(t)-x_{j}^{m}(t)\right)\right] u_{j k}^{m}(t) d t=0,
\end{aligned}
$$

where the second integral converges to zero by the weak convergence of the controls, and the third integral converges to zero by the uniform convergence of the states and the $L^{\infty}$ bound on the controls. Hence, for all $v \in C_{c}^{\infty}[0, T]$,

$$
\begin{aligned}
\int_{0}^{T} \frac{d v}{d t} x^{*}(t) d t & =\lim _{m \rightarrow \infty} \int_{0}^{T} \frac{d v}{d t} x_{j}^{m}(t) d t \\
& =-\lim _{m \rightarrow \infty} \int_{0}^{T} v\left[\omega_{j}+\frac{1}{N} \sum_{k=1}^{N} u_{j k}^{m}(t) \sin \left(x_{k}^{m}(t)-x_{j}^{m}(t)\right)\right] d t \\
& =-\int_{0}^{T} v(t)\left[\omega_{j}+\frac{1}{N} \sum_{k=1}^{N} u_{j k}^{*}(t) \sin \left(x_{k}^{*}(t)-x_{j}^{*}(t)\right)\right] d t
\end{aligned}
$$

where the first equality follows from the uniform convergence of the states, the second via integration by parts, and the final equality follows from the previous note.

By the uniform convergence of the states, we also have that

$$
\frac{d x_{N+1}^{*}}{d t}=\frac{1}{N^{2}} \sum_{i, j} \cos \left(x_{i}^{*}(t)-x_{j}^{*}(t)\right) .
$$

Next we show that for $i, j \in\{1,2, \ldots, N\}$, ess $\sup \left(u_{i j}^{*}\right) \leq M$. Assume toward a contradiction that there exist $i, j \in\{1,2, \ldots, N\}$, so that ess $\sup \left(u_{i j}^{*}\right)>M$. Then, there exists a set $E$ with $\mu(E)>0$, so that for $t \in E, u_{i j}^{*}(t)>M$. Hence, by the weak convergence of the controls,

$$
\mu(E) M<\int_{E} u_{i j}^{*}(t) d t=\lim _{m \rightarrow \infty} \int_{E} u_{i j}^{m}(t) d t \leq \lim _{m \rightarrow \infty} \mu(E) M=\mu(E) M,
$$

a contradiction. Thus, we conclude that for $i, j \in\{1,2, \ldots, N\}$, ess $\sup \left(u_{i j}^{*}\right) \leq M$. 
Finally, since the $L^{2}$ norm is lower semicontinuous with respect to weak convergence,

$$
J_{1}\left(u^{*}\right)=\int_{0}^{T} \sum_{j, k} u_{j k}^{*}(t)^{2} d t \leq \underline{\lim } \int_{0}^{T} \sum_{j, k} u_{j k}^{m}(t)^{2} d t=\min _{u \in U_{a d}} J_{1}(u) .
$$

Thus, $u^{*}$ is optimal, and the desired existence result is proved.

In order to characterize the optimal coupling strengths, we use the right hand sides of the differential equations for the states to define a vector valued function $g(x, u):\left[\Re^{N+1}, \Re^{N \times N}\right] \rightarrow \Re^{N+1}$ as

$$
g(x, u):=\left(\frac{d x_{1}}{d t}(x, u), \ldots, \frac{d x_{N+1}}{d t}(x, u)\right)^{\prime},
$$

where' denotes the transpose. In addition, we define a function $f(x, u)$ by $f(x, u):=\sum_{i=1}^{N} u_{i j}^{2}$. In terms of $g$ and $f$ the Hamiltonian function, $H$ : $\Re^{N+1} \times \Re^{N \times N} \times \Re^{N+1} \times \Re \rightarrow \Re$, is given by

$$
\begin{aligned}
H\left(x, u, y, y_{0}\right) & =y^{\prime} g(x, u)+y_{0} f(x, u) \\
& =\sum_{i=1}^{N} y_{i}\left(\omega_{i}+\frac{1}{N} \sum_{j} u_{i j} \sin \left(x_{j}-x_{i}\right)\right) \\
& +\frac{y_{N+1}}{N^{2}}\left[\left(\sum_{j} \sin x_{j}\right)^{2}+\left(\sum_{j} \cos x_{j}\right)^{2}\right]+y_{0} \sum_{i, j} u_{i j}^{2} .
\end{aligned}
$$

The following theorem, adapted from [13], characterizes the solutions of Problem I.

Theorem 2.2. If $\left(x^{*}, u^{*}\right)$ is a solution to Problem I, then there exists a pair $\left(y_{0}, y(t)\right)$ so that the following conditions are met:

(i) The map $y:[0, T] \rightarrow \Re^{N+1}$ is absolutely continuous;

(ii) $y_{0} \in \Re$ and $y_{0} \leq 0$;

(iii) $\left(y_{0}, y(t)\right) \neq(0,0)$ for every $t \in[0, T]$;

(iv) the adjoint differential equation holds, that is

$$
\frac{d y}{d t}=-\frac{\partial H}{\partial x}\left(x^{*}(t), u^{*}, y(t), y_{0}\right)
$$


(v) the Hamiltonian maximization condition holds, that is

$$
H\left(x^{*}(t), u^{*}(t), y(t), y_{0}\right)=\max _{u \in[-M, M]^{N \times N}}\left\{H\left(x^{*}(t), u(t), y(t), y_{0}\right)\right\}
$$

(vi) and the transversality condition $y_{i}(T)=0$ holds for $i=1, \ldots, N$.

Note that the adjoint equations may be written out componentwise:

$$
\begin{aligned}
\frac{d y_{i}}{d t} & =\frac{1}{N} \sum_{j \neq i, j=1}^{N}\left(y_{i}(t) u_{i j}^{*}(t)-y_{j}(t) u_{j i}^{*}(t)\right) \cos \left(x_{j}^{*}(t)-x_{i}^{*}(t)\right) \\
& -\frac{2 y_{N+1}(t)}{N^{2}} \sum_{j=1}^{N} \sin \left(x_{j}^{*}(t)-x_{i}^{*}(t)\right) \quad \text { for } i \in\{1,2, \ldots, N\} \\
\frac{d y_{N+1}}{d t} & =0 .
\end{aligned}
$$

In particular, $y_{N+1}$ is constant.

Since the boundary conditions, $x_{N+1}(0)=0$ and $x_{N+1}(T)=A$, make Problem I overdetermined, it is possible that the adjoint variable $y_{0}$ is equal to zero, so that the objective functional does not contribute to the necessary conditions. If $y_{0}=0$, then the Hamiltonian is linear in each $u_{i j}$ so that each optimal coupling strength is either bang-bang or singular. If, on the other hand, $y_{0} \neq 0$, then the quadratic form of the objective functional implies that $u_{i j}$ is continuous. Theorem 2.2 will now be used to show that, under certain conditions, $y_{0} \neq 0$.

Theorem 2.3. If the following conditions are met, then $y_{0} \neq 0$.

(i) The oscillators are not identical. That is, for some $i$ and $j, \omega_{i} \neq \omega_{j}$.

(ii) $M>\sqrt{\frac{T N(N-1)}{A}} K$.

Proof. We show that $y_{0} \neq 0$ by showing that $y_{0}=0$ contradicts the optimality of $u^{*}$. Specifically, let $S:=\left\{t: r^{2}(t) \neq 0\right\}$, and note that since $0 \leq r^{2}(t) \leq 1$ and $\int_{0}^{T} r^{2}(t) d t=A$, the measure of $S$ is greater than or equal to $A$. We claim that, if $y_{0}=0$, then for almost every $t \in S$, $\max _{i j \in\{1,2 \ldots, N\}}\left|u_{i j}^{*}(t)\right|=M$. Once this claim is established, we can conclude 
that $y_{0} \neq 0$ since, according to the claim and condition $(i i)$ of this theorem, if $y_{0}=0$ then:

$$
\int_{0}^{T} \sum_{i j}\left(u_{i j}^{*}(t)\right)^{2} d t \geq \int_{S} \sum_{i j}\left(u_{i j}^{*}(t)\right)^{2} d t \geq A M^{2}>T N(N-1) K^{2}=\int_{0}^{T} \sum_{i \neq j} K^{2} d t
$$

which contradicts the optimality of $u^{*}(t)$ (since the constant control, $u_{i j} \equiv K$ for $i \neq j$ is admissible by design).

Suppose that $y_{0}=0$. Then the Hamiltonian becomes

$$
\begin{aligned}
H\left(x^{*}(t), u^{*}(t), y(t), 0\right) & =\sum_{i} y_{i}(t)\left(\omega_{i}+\frac{1}{N} \sum_{j} u_{i j}^{*}(t) \sin \left(x_{j}^{*}(t)-x_{i}^{*}(t)\right)\right) \\
& +\frac{y_{N+1}}{N^{2}} \sum_{i j} \cos \left(x_{j}^{*}(t)-x_{i}^{*}(t)\right) .
\end{aligned}
$$

By (13) and the Maximum Principle, given any $i, j \in\{1,2, \ldots, N\}$ such that $i \neq j$, if $y_{i}(t) \sin \left(x_{j}^{*}(t)-x_{i}^{*}(t)\right) \neq 0$, then $u_{i j}^{*}(t)= \pm M$. Hence, in order to establish the claim, it suffices to show that at almost every point $t \in S$ there exits $i, j \in\{1,2, \ldots, N\}$ with $i \neq j$, such that $y_{i}(t) \sin \left(x_{j}^{*}(t)-x_{i}^{*}(t)\right) \neq 0$.

Suppose toward a contradiction that there exists an open interval $E \subset$ $S$ such that for all $t \in E$, and for all $i, j \in\{1,2, \ldots, N\}$ with $i \neq j$, $y_{i}(t) \sin \left(x_{j}^{*}(t)-x_{i}^{*}(t)\right)=0$. Then at each point $t \in E$, and for all $i, j \in$ $\{1,2, \ldots, N\}$ such that $i \neq j$, either $y_{i}(t)=0$ or $\sin \left(x_{j}^{*}(t)-x_{i}^{*}(t)\right)=0$. In fact, for all $i \in\{1,2, \ldots, N\}, y_{i}(t) \equiv 0$ in $E$. To see this, note that if there exists $i \in\{1, \ldots, N\}$ and $t^{*} \in E$ so that $y_{i}\left(t^{*}\right) \neq 0$, then, since $y_{i}$ is continuous, there exists an open interval $I \in E$ so that $t^{*} \in I$, and $y_{i}(t) \neq 0$ in $I$. Hence for all $j \in\{1,2, \ldots, N\}$ such that $i \neq j, \sin \left(x_{j}^{*}(t)-x_{i}^{*}(t)\right) \equiv 0$ in $I$. It follows that for all $j, k \in\{1,2, \ldots, N\}$ with $j, k \neq i, x_{j}^{*}(t)-x_{i}^{*}(t)$, and thus $x_{k}^{*}(t)-x_{j}^{*}(t)$, is a constant multiple of $\pi$ in $I$. Thus, for all $j, k \in\{1,2, \ldots, N\}$,

$$
0 \equiv \frac{d\left(\sin \left(x_{j}^{*}(t)-x_{k}^{*}(t)\right)\right)}{d t}=\cos \left(x_{j}^{*}(t)-x_{k}^{*}(t)\right)\left(\omega_{j}-\omega_{k}\right)= \pm\left(\omega_{j}-\omega_{k}\right)
$$

in $I$, which contradicts assumption $(i)$ of this theorem. Therefore, it must be that for all $i \in\{1, \ldots, N\}, y_{i}(t) \equiv 0$ in $E$. Hence, by condition (iii) of Theorem 2.2, $y_{N+1} \neq 0$. Since for all $i \in\{1,2, \ldots, N\}, y_{i}(t) \equiv 0$ in $E$, for all $i \in\{1,2, \ldots, N\}$, condition (iv) of Theorem 2.2 implies

$$
0 \equiv \frac{d y_{i}}{d t}=-\frac{2 y_{N+1}}{N^{2}} \sum_{j} \sin \left(x_{j}^{*}(t)-x_{i}^{*}(t)\right) .
$$


in $E$. Since $y_{N+1} \neq 0$, it follows that for all $i \in\{1,2, \ldots, N\}$

$$
\begin{aligned}
0 & \equiv \sum_{j} \sin \left(x_{j}^{*}(t)-x_{i}^{*}(t)\right) \\
& =\cos x_{i}^{*}(t) \sum_{j} \sin x_{j}^{*}(t)-\sin x_{i}^{*}(t) \sum_{j} \cos x_{j}^{*}(t) \\
& =a(t) \cos x_{i}^{*}(t)-b(t) \sin x_{i}^{*}(t)
\end{aligned}
$$

in $E$, where $a(t)=\sum_{j} \sin x_{j}^{*}(t)$, and $b(t)=\sum_{j} \cos x_{j}^{*}(t)$. Since $r^{2}(t) \neq 0$ in $E$, it cannot be that both $a(t)$ and $b(t)$ are equal to zero. Hence, by (16), for $i \in\{1,2, \ldots, N\}$ and $t \in E$, the vector $\left(\cos x_{i}^{*}(t), \sin x_{i}^{*}(t)\right)$ belongs to the one dimensional vector space spanned by the vector $(b(t), a(t))$. Thus, at each point $t \in E$ and for all $i, j \in\{1,2, \ldots, N\}$ there exists a real number $\alpha_{j}(t)$ so that

$$
\left(\cos x_{i}^{*}(t), \sin x_{i}^{*}(t)\right)=\alpha_{j}(t)\left(\cos x_{j}^{*}(t), \sin x_{j}^{*}(t)\right) .
$$

Equation (17) implies that at each point $t \in E$ and for all $i, j \in\{1,2, \ldots, N\}$, $x_{i}(t) \equiv x_{j}(t) \bmod \pi$. Hence for all $i, j \in\{1,2, \ldots, N\}, \sin \left(x_{j}(t)-x_{i}(t)\right) \equiv 0$ in $E$, which contradicts assumption $(i)$ of this theorem. Therefore, there exists no open interval $E \subset S$ such that, for all $i, j \in\{1,2, \ldots, N\}$ with $i \neq j$, $y_{i}(t) \sin \left(x_{j}^{*}(t)-x_{i}^{*}(t)\right) \equiv 0$ in $E$. It follows from the continuity of the states and the adjoints that for almost every $t \in S$ there exists $i, j \in\{1, \ldots, N\}$ with $i \neq j$, so that $y_{i}(t) \sin \left(x_{j}^{*}(t)-x_{i}^{*}(t)\right) \neq 0$. Thus, for almost every $t \in S$, there exists $i, j \in\{1, \ldots, N\}$ with $i \neq j$, so that $\left|u_{i j}^{*}(t)\right|=M$. Therefore, it must be that $y_{0} \neq 0$ and the theorem is established.

Since we have shown that $y_{0} \neq 0$, taking $y_{0}=-1$ we can characterize an optimal control by maximizing the Hamiltonian pointwise along the optimal trajectory:

$$
u_{i j}^{*}(t)=\max \left(-M, \min \left(\frac{y_{i}(t) \sin \left(x_{j}^{*}(t)-x_{i}^{*}(t)\right)}{2 N}, M\right)\right) .
$$

Note that the condition $y_{0} \neq 0$ implies that the coupling strengths will not switch between two states $\pm M$, but vary smoothly.

\section{Maximizing the Synchrony of a Network with Bounded Connec- tivity Cost}

In this section, given the initial phases and natural velocities of a set of oscillators, $\left(x_{0}\right.$ and $\left.\omega\right)$, we will find the coupling strengths $u_{i j}$ that enable the 
set of oscillators to maximize their synchrony while keeping the connectivity cost below a prescribed bound. Later, we will compare the performance of the time varying network to that of the classical Kuramoto network. For this purpose, we set the prescribed bound on the connectivity cost equal to that of a Kuramoto network with constant coupling strength $K$. Specifically, given $x_{0}, \omega$, and $K$, we will find the coupling strengths, $u_{i j}^{*}(t)$, that maximize

$$
J_{2}(u):=\int_{0}^{T} r^{2}(t) d t
$$

subject to

$$
\int_{0}^{T}\left(u_{i j}^{*}(t)\right)^{2} d t=C:=T N(N-1) K^{2}
$$

As before, the control set $U$ is defined as $U=[-M, M]^{N \times N}$, i.e. the set of all matrices with elements in the closed interval $[-M, M]$. Controls for this problem are functions $u:[0, T] \rightarrow U$, such that each component, $u_{i j}$, is a Lebesgue measurable function from $[0, T]$ to $[-M, M]$. To incorporate the constraint $\int_{0}^{T} \sum_{i j} u_{i j}^{2} d t \leq C$ into the problem, we introduce an artificial state variable $x_{N+1}$, which satisfies:

$$
\begin{array}{r}
\frac{d x_{N+1}}{d t}=\sum_{i j} u_{i j}^{2}(t) ; \\
x_{N+1}(0)=0, x_{N+1}(T) \leq C .
\end{array}
$$

We say that a vector of states $x(t)=\left(x_{1}(t), \ldots, x_{N}(t), x_{N+1}(t)\right)$ corresponds to the control $u$ if its components are absolutely continuous functions which satisfy

$\frac{d x_{i}}{d t}=\omega_{i}+\frac{1}{N} \sum_{j=1}^{N} u_{i j}(t) \sin \left(x_{j}(t)-x_{i}(t)\right) ; \quad x_{i}(0)=x_{i}^{0} \quad$ for $i \in\{1,2, \ldots, N\}$,

and

$$
\frac{d x_{N+1}}{d t}=\sum_{i, j=1}^{N} u_{i j}^{2}(t) ; \quad x_{N+1}(0)=0 .
$$

In this case, we call $(x(t), u(t))$ a trajectory-control pair.

The set of admissible controls, $U_{a d}$, is defined as follows: Let $(x(t), u(t))$ be a trajectory-control pair, with $u:[0, T] \rightarrow U$. We will say that $u \in U_{a d}$ 
if $x_{N+1}(T) \leq C$. Note that $U_{a d} \neq \emptyset$ because the control $u_{i j}(t) \equiv K$ is an element of $U_{a d}$ by design.

With this framework the problem may be stated as follows:

Problem II:

Find a trajectory-control pair $\left(x^{*}, u^{*}\right)$ so that $u^{*} \in U_{a d}$, and

$$
J_{2}\left(u^{*}\right)=\max _{u \in U_{a d}} J_{2}(u)
$$

Theorem 3.1. There exists a solution, $\left(x^{*}, u^{*}\right)$, to Problem II.

As in Problem I, the right hand sides of the differential equations for the states can be used to define $g(x, u):\left[\Re^{N+1}, \Re^{N \times N}\right] \rightarrow \Re^{N+1}$. In addition, we define a new function $f(x, u)$, corresponding to the integrand of the objective functional of Problem II, by

$$
f(x, u):=\frac{1}{N^{2}}\left[\left(\sum_{j} \sin x_{j}\right)^{2}+\left(\sum_{j} \cos x_{j}\right)^{2}\right]
$$

and the Hamiltonian function $H: \Re^{N+1} \times \Re^{N \times N} \times \Re^{N+1} \times \Re \rightarrow \Re$ by

$$
\begin{aligned}
H\left(x, u, y, y_{0}\right) & =y^{\prime} g(x, u)+y_{0} f(x, u) \\
& =\sum_{i=1}^{N} y_{i}\left(\omega_{i}+\frac{1}{N} \sum_{j} u_{i j} \sin \left(x_{j}-x_{i}\right)\right) \\
& +y_{N+1} \sum_{i, j} u_{i j}^{2} \\
& +\frac{y_{0}}{N^{2}}\left[\left(\sum_{j} \sin x_{j}\right)^{2}+\left(\sum_{j} \cos x_{j}\right)^{2}\right] .
\end{aligned}
$$

The following theorem, adapted from [13], characterizes the solutions of Problem II.

Theorem 3.2. If $\left(x^{*}, u^{*}\right)$ is a solution to Problem II, then there exists a pair $\left(y_{0}, y(t)\right)$ so that the following conditions are met:

(i) The map $y:[0, T] \rightarrow \Re^{N+1}$ is absolutely continuous;

(ii) $y_{0} \in \Re$ and $y_{0} \geq 0$; 
(iii) $\left(y_{0}, y(t)\right) \neq(0,0)$ for every $t \in[0, T]$;

(iv) the adjoint differential system holds, that is

$$
\frac{d y}{d t}=-\frac{\partial H}{\partial x}\left(x^{*}(t), u^{*}(t), y(t), y_{0}\right)
$$

(v) the Hamiltonian maximization condition holds, that is

$$
H\left(x^{*}(t), u^{*}(t), y(t), y_{0}\right)=\max _{u \in U}\left\{H\left(x^{*}(t), u, y(t), y_{0}\right)\right\}
$$

(vi) and the transversality condition $y_{i}(T)=0$ holds for $i=1, \ldots, N$.

The adjoint equations may be written out componentwise:

$$
\begin{aligned}
\frac{d y_{i}}{d t} & =\frac{1}{N} \sum_{j \neq i, j=1}^{N}\left(y_{i}(t) u_{i j}^{*}(t)-y_{j}(t) u_{j i}^{*}(t)\right) \cos \left(x_{j}^{*}(t)-x_{i}^{*}(t)\right) \\
& -\frac{2 y_{0}(t)}{N^{2}} \sum_{j=1}^{N} \sin \left(x_{j}^{*}(t)-x_{i}^{*}(t)\right) \text { for } i \in\{1,2, \ldots, N\} \\
\frac{d y_{N+1}}{d t} & =0 .
\end{aligned}
$$

In particular, we see that $y_{N+1}$ is constant.

As in the previous problem, Theorem 3.2 can be used to show that if $M$ is large enough and the oscillators are not identical, then $y_{N+1} \neq 0$. This allows us to characterize the optimal control in terms of the adjoint and state variables by setting the derivative of the Hamiltonian with respect to each coupling strength to zero.

Theorem 3.3. Let $A$ be the synchrony obtained by an identical system of oscillators governed by the mean-field control $u_{i j} \equiv K$. If the following conditions are met, $y_{N+1} \neq 0$.

(i) $A \neq 0$.

(ii) The oscillators are not identical,i.e., for some $i$ and $j, \omega_{i} \neq \omega_{j}$.

(iii) $M>\sqrt{\frac{C}{A}}$. 
In summary, if an identical set of oscillators coupled via the admissible Kuramoto network $u_{i j} \equiv K$ for $i \neq j$ has nonzero order, if there is some variability in the oscillators' natural velocities, and if $M$ is large enough, then $y_{0} \neq 0$. Note that the first condition is always satisfied if the order parameter is initially nonzero.

The proof of Theorem 3.3 is like that of Theorem 2.3, but with $y_{N+1}$ in place of $y_{0}$.

Taking $y_{N+1}=1$, the Hamiltonian maximum condition allows us to characterize the optimal control:

$$
u_{i j}^{*}=\max \left(-M, \min \left(\frac{-y_{i}(t) \sin \left(x_{j}^{*}-x_{i}^{*}\right)}{2 N}, M\right)\right) .
$$

The assumptions of Theorem 3.3 along with Theorem 3.2 can be used to show that $x_{N+1}^{*}(T)=C$, i.e. the optimal control obtains the maximum allowed connectivity cost. In particular, we have the following theorem:

Theorem 3.4. Let $\left(x^{*}, u^{*}\right)$ be an optimal trajectory control pair. If

(i) $u^{*}$ is not identically equal to zero, and

(ii) controls with $\left|u_{i j}(t)\right| \equiv M$ for all $i \neq j$ are not admissible, that is,

$$
\int_{0}^{T} \sum_{i j} u_{i j}^{2}(t) d t=T N(N-1) M^{2}>C,
$$

and if, in addition, the conditions of Theorem 3.3 hold, $x_{N+1}^{*}(T)=C$.

With respect to assumption $(i)$ of this theorem, it is easy to show that $u^{*} \equiv 0$ is not optimal, and assumption $(i i)$ of this theorem is satisfied if $M$ is large enough.

Proof. Suppose that the assumptions of this theorem are met. Then there exists $\epsilon_{0}>0$ so that $x_{N+1}^{*}(T)=C-\epsilon_{0}$, and by Theorem 3.3 and Theorem $3.2, u^{*}$ is continuous. Since $u^{*}$ is continuous and not identically equal to 0 , $M$, or $-M$, we may assume (without loss of generality) that there exists an open interval $I$ and $i \neq j$, so that for all $t \in I, \epsilon_{1}<u_{i j}^{*}(t)<M-\epsilon_{1}$. Thus the control variation $u^{\epsilon}$ defined by

$$
u_{i j}^{\epsilon}(t)=u_{i j}^{*}(t)+\epsilon ; \quad \text { for } t \in I,
$$




$$
\begin{gathered}
u_{i j}^{\epsilon}(t)=u_{i j}^{*}(t) ; \quad \text { for } t \notin I \\
u_{k l}^{\epsilon}(t) \equiv u_{k l}^{*}(t) ; \quad \text { for }(k, l) \neq(i, j)
\end{gathered}
$$

is admissible for $\epsilon$ small enough. Let $g(x, u)$ and $f(x, u)$ be defined as in Theorem 3.1. Then, following the development of the necessary conditions presented by Lenhart and Workman [14],

$y_{0} J_{2}\left(u^{\epsilon}\right)=\int_{0}^{T} y_{0} f\left(x^{\epsilon}, u^{\epsilon}, t\right)+\frac{d}{d t}\left(y(t)^{\prime} x^{\epsilon}(t)\right) d t+y(0)^{\prime} x^{\epsilon}(0)-y(T)^{\prime} x^{\epsilon}(T)$.

Taking the limit of $\frac{J_{2}\left(x^{\epsilon}, u^{\epsilon}\right)-J_{2}\left(x^{*}, u^{*}\right)}{\epsilon}$ as $\epsilon \rightarrow 0$ we see that

$$
\begin{aligned}
0 & =\left.\frac{d}{d \epsilon} y_{0} J_{2}\left(u^{\epsilon}\right)\right|_{\epsilon=0} \\
& =\int_{0}^{T}\left(y_{0} f_{u_{i j}}\left(t, x^{*}(t), u^{*}(t)\right)+y(t)^{\prime} g_{u_{i j}}\left(t, x^{*}(t), u^{*}(t)\right)\right) h(t) d t \\
& -\left.y_{N+1}(T) \frac{\partial x_{N+1}^{\epsilon}}{\partial \epsilon}(T)\right|_{\epsilon=0}=-y_{N+1} \int_{I} 2 u_{i j}(t) d t .
\end{aligned}
$$

The first equality follows from the optimality of $u^{*}$. The second equality follows from the chain rule, the adjoint equations, $y_{i}(T)=0$ for $i=1,2, \ldots, N$, and $u_{k l}^{\epsilon}=u_{k l}^{*}$ if $(k, l) \neq(i, j)$. The third equality follows from that fact that

$y_{0} f_{u_{i j}}\left(t, x^{*}(t), u^{*}(t)\right)+y(t)^{\prime} g_{u_{i j}}\left(t, x^{*}(t), u^{*}(t)\right)=H_{u_{i j}}\left(t, x^{*}(t), y(t), u^{*}(t)\right)=0$

in $I$. Since $u_{i j}(t)>0$ on $I,(25)-(27)$ imply that $y_{N+1}=0$, which contradicts Theorem 3.3. Thus, it must be that $x_{N+1}^{*}(T)=C$.

\section{Balancing Network Synchrony and Connectivity Cost}

In this section, we consider an intermediate problem. Here we will find the coupling strengths that maximize the difference between the network synchrony and a weighted connectivity cost:

$$
J_{3}(u):=\int_{0}^{T} r^{2}(t) d t-B \int_{0}^{T} \sum_{i j} u_{i j}^{2}(t) d t .
$$

As before, the control set $U$ is defined as $U=[-M, M]^{N \times N}$. Controls for this problem are functions $u:[0, T] \rightarrow U$, such that each component $u_{i j}$ is a 
Lebesgue measurable function from $[0, T]$ to $[-M, M]$. We say that a vector of state trajectories $x(t)=\left(x_{1}(t), \ldots, x_{N}(t)\right)$ corresponds to the control $u(t)$, if the components of $x(t)$ are absolutely continuous functions that satisfy

$$
\frac{d x_{i}}{d t}=\omega_{i}+\frac{1}{N} \sum_{j=1}^{N} u_{i j}(t) \sin \left(x_{j}-x_{i}\right) ; \quad x_{i}(0)=x_{i}^{0} \quad \text { for } i \in\{1,2, \ldots, N\} .
$$

In this case, we call $(x, u)$ a trajectory-control pair. Within this framework, the problem may be stated as follows:

Problem III:

Find a trajectory-control pair $\left(x^{*}, u^{*}\right)$ so that $u^{*}(t) \in U$ for $t \in[0, T]$, and

$$
J_{3}\left(u^{*}\right)=\max _{u \in U} J_{3}(u) .
$$

Note that this problem is simpler than the previous two because it does not have any final time constraints.

Theorem 4.1. There exists a solution, $\left(x^{*}, u^{*}\right)$, to Problem III.

The proof of Theorem 4.1 is similar to the proofs of existence of an optimal control in the previous two problems.

The characterization of the optimal control follows from Pontryagin's Maximum Principle [14].

Theorem 4.2. Given an optimal control $u$, there exists $N$ adjoint variables, $y_{i}$, such that the Hamiltonian:

$$
\begin{aligned}
H(t, x, y, u) & =\frac{1}{N^{2}}\left[\left(\sum_{j} \sin x_{j}\right)^{2}+\left(\sum_{j} \cos x_{j}\right)^{2}\right]-B \sum_{i, j} u_{i j}^{2} \\
& +\sum_{i} y_{i}\left(\omega_{i}+\frac{1}{N} \sum_{j} u_{i j} \sin \left(x_{j}-x_{i}\right)\right)
\end{aligned}
$$

is constant along the optimal trajectory and is maximized pointwise by the optimal control. In addition, for $i=1 \ldots N$ :

$\frac{d y_{i}}{d t}=\frac{1}{N} \sum_{j=1}^{N}\left(y_{i} u_{i j}-y_{j} u_{j i}\right) \cos \left(x_{j}-x_{i}\right)-\frac{2}{N^{2}} \sum_{j=1}^{N} \sin \left(x_{j}-x_{i}\right) ; \quad y_{i}(T)=0$. 
This theorem allows us to characterize an optimal control in terms of the corresponding states and adjoints:

$$
u_{i j}^{*}=\max \left(-M, \min \left(\frac{y_{i}(t) \sin \left(x_{j}-x_{i}\right)}{2 B N}, M\right)\right) .
$$

For this problem, it can be shown that the optimality system has a unique solution for $T$ sufficiently small. Hence, the necessary conditions are also sufficient and the optimal control is unique.

Theorem 4.3. For $T$ sufficiently small, there exists a unique optimal control.

Proof. We show that the optimal control is unique by showing that the optimality system has a unique solution.

Given two solutions $(x, y)$ and $(\bar{x}, \bar{y})$ to the optimality system, make the change of variables $x_{i}=\mathrm{e}^{k t} v_{i}$ and $y_{i}=\mathrm{e}^{-k t} w_{i}$. Then $v_{i}$ satisfies:

$$
\begin{aligned}
& \frac{1}{2}\left(v_{i}(t)-\bar{v}_{i}(t)\right)^{2}+k \int_{0}^{t}\left(v_{i}-\bar{v}_{i}\right)^{2} d t= \\
& \frac{1}{N} \int_{0}^{T} \mathrm{e}^{-k t}\left[\sum_{j} u_{i j} \sin \left(x_{j}-x_{i}\right)-\bar{u}_{i j} \sin \left(\bar{x}_{i}-\bar{x}_{j}\right)\right]\left(v_{i}-\bar{v}_{i}\right) d t .
\end{aligned}
$$

Applying Jensen's inequality to the right hand side of (28) and using $\left|u_{i j}\right| \leq$ $M$, we find that

$\frac{1}{2}\left(v_{i}(t)-\bar{v}_{i}(t)\right)^{2}+k \int_{0}^{t}\left(v_{i}-\bar{v}_{i}\right)^{2} d t \leq 4 M^{2}+\frac{5}{2}\left\|v_{i}-\bar{v}_{i}\right\|_{L^{2}}^{2}+\frac{2}{N} \sum\left\|v_{j}-\bar{v}_{j}\right\|_{L^{2}}^{2}$.

Meanwhile $w_{i}$ satisfies:

$$
\begin{aligned}
& \frac{1}{2}\left(w_{i}(t)-\bar{w}_{i}(t)\right)^{2}+k \int_{t}^{T}\left(w_{i}-\bar{w}_{i}\right)^{2} d t= \\
& \frac{1}{N} \int_{t}^{T} \mathrm{e}^{k t}\left(\sum_{j}\left(y_{i} u_{i j}-y_{j} u_{j i}\right) \cos \left(x_{j}-x_{i}\right)-\left(\bar{y}_{i} \bar{u}_{i j}-\bar{y}_{j} \bar{u}_{j i}\right) \cos \left(\bar{x}_{i}-\bar{x}_{j}\right)\right)\left(w_{i}-\bar{w}_{i}\right) d t \\
& -\frac{2}{N^{2}} \int_{t}^{T} \mathrm{e}^{k t}\left(\sum_{j} \sin \left(x_{j}-x_{i}\right)-\sin \left(\bar{x}_{j}-\bar{x}_{i}\right)\right)\left(w_{i}-\bar{w}_{i}\right) d t
\end{aligned}
$$


Since the adjoint vector is the solution of a linear differential equation with bounded coefficients, its components are bounded on $[0, T]$ by some constant $C_{0}$. Hence, applying Jensen's inequality to the right hand side of (30) and using $\left|u_{i j}\right| \leq M$, and $\left|y_{i}\right| \leq C_{0}$ we find that,

$$
\begin{aligned}
& \frac{1}{2}\left(w_{i}(t)-\bar{w}_{i}(t)\right)^{2}+k \int_{t}^{T}\left(w_{i}-\bar{w}_{i}\right)^{2} d t \leq \\
& {\left[\frac{3}{2}+4 M^{2}\right]\left\|w_{i}-\bar{w}_{i}\right\|_{L^{2}}^{2}+\frac{4 M^{2}}{N} \sum_{j}\left\|w_{j}-\bar{w}_{j}\right\|_{L^{2}}^{2}+} \\
& {\left[8 M^{2} C_{0}^{2}+\frac{2}{N^{2}}\right] \mathrm{e}^{4 k T}\left\|v_{i}-\bar{v}_{i}\right\|_{L^{2}}^{2}+} \\
& {\left[8 M^{2} C_{0}^{2}+\frac{2}{N^{3}}\right] \mathrm{e}^{4 k T} \sum_{j}\left\|v_{j}-\bar{v}_{j}\right\|_{L^{2}}^{2}+8 M^{2} C_{0}^{2} T \mathrm{e}^{4 k T} .}
\end{aligned}
$$

Integrating (29) and (31) from 0 to $T$ and summing over $i$ we find that

$\sum_{i}\left\|w_{i}-\bar{w}_{i}\right\|_{L^{2}}^{2}+\left\|v_{i}-\bar{v}_{i}\right\|_{L^{2}}^{2} \leq T\left[C_{1} \sum\left\|w_{i}-\bar{w}_{i}\right\|_{L^{2}}^{2}+C_{2} \sum\left\|v_{i}-\bar{v}_{i}\right\|_{L^{2}}^{2}+C_{3}\right]$,

where the constants on the left hand side of the inequality are bounded if $T$ is sufficiently small. Hence for $T$ small, the solution of the optimality system is unique.

\section{Numerical Simulations}

Having derived necessary conditions for the optimal coupling strengths for each of the three problems, we used a forward-backward sweep algorithm [14] to find coupling strengths that satisfy the optimality system. Briefly, an initial control and the state and adjoint differential equations are used to solve for the state and adjoint variables. The necessary conditions are then used to update the control variable. A convex combination of the old and updated control is then used in the subsequent iteration of the routine. Our convergence criteria stops the routine in case the cumulative, absolute or point wise, relative error is small for both the states and controls. Specifically, in terms of the controls, the absolute, point-wise and relative, cumulative criteria correspond to, for all $i, j=1, \ldots, N, \max _{t}\left|u_{i j}^{k}-u_{i j}^{k+1}\right|<\epsilon$ and for all $i, j=1, \ldots, N, \frac{\int_{0}^{T}\left|\left(u_{i j}^{k}-u_{i j}^{k+1}\right)\right| d t}{\left.\int_{0}^{T} \mid u_{i j}^{k+1}\right) \mid d t}<\epsilon$, respectively, where superscripts 
are used to denote the number of iterations. Convergence of the routine can be problematic for larger networks or longer time intervals. In these cases, we observe that convex combinations that weigh previous controls more heavily can aid in convergence. In order obtain approximate solutions of the optimality system, we have used $\epsilon$ on the order of $10^{-6}-10^{-3}$ in implementing the convergence criteria.

As we are interested in investigating the potential benefits of optimizing network structure and dynamics for synchrony, our parameter choices are guided by the behavior of the classical Kuramoto model. In particular, in case the parameters are chosen so that the order parameter of the classical Kuramoto model oscillates, the vector of initial conditions is taken as $x_{0}=$ $[0, \ldots, 0]$, in an attempt to minimize transience. In case the parameters are chosen so that the order parameter of the classical Kuramoto model is constant, the vector of initial conditions is chosen as a phase-locked solution. We note that, in general, the solution depends on all of the parameters involved, including the initial phases, the natural velocities, the coupling strength, and the final time. In this paper, however, we do not investigate these dependencies in detail. Instead, we consider some simple cases, in order to illustrate salient features and characterize the potential benefits of time-varying networks.

Initially, we consider Problem I. Specifically, we seek controls that minimize the cost of achieving a prescribed level of synchrony for a network of two oscillators with $\omega=<0,10>$. For this choice of $\omega$, the order parameter is constant for $K \geq 10$ and oscillatory for $K<10$. Using the necessary conditions, we search for optimal controls for various values of $K$, using $u_{i j} \equiv K$ for $i \neq j$ to initialize the numerical routine. Numerical solutions of the optimality system undergo a qualitative change at $K=10$. In particular, for $K \leq 10$ the algorithm converges to a solution with oscillatory coupling strengths, while for $K>10$ the algorithm converges to a solution with nearly constant coupling strengths. (Note that, in general, solutions of the problem are not constant due to the integral form of the objective functional and the effect of the finite final time.) For $K \leq 10$, the optimal coupling strengths are considerably more efficient than the constant coupling strengths at achieving a prescribed level of order. The control pictured in Figure 1 achieves the same order as a Kuramoto network with $K=5$. Its coupling cost, however, is equal to that of a Kuramoto network with $K=2.3637$. In another simulation of the same oscillator pair, a time-varying controlled network achieved the same level of synchrony as a Kuramoto network with $K=10$ for a cou- 
pling cost that is equivalent to that of a Kuramoto network with $K=3.7248$. As $K$ increases above 10, the solutions of the optimality system appear to converge to nearly constant solutions. For example, in a simulation with $K=20$, the controlled network is close to constant and has a coupling cost that is equivalent to that of a Kuramoto network with $K=19.8651$.
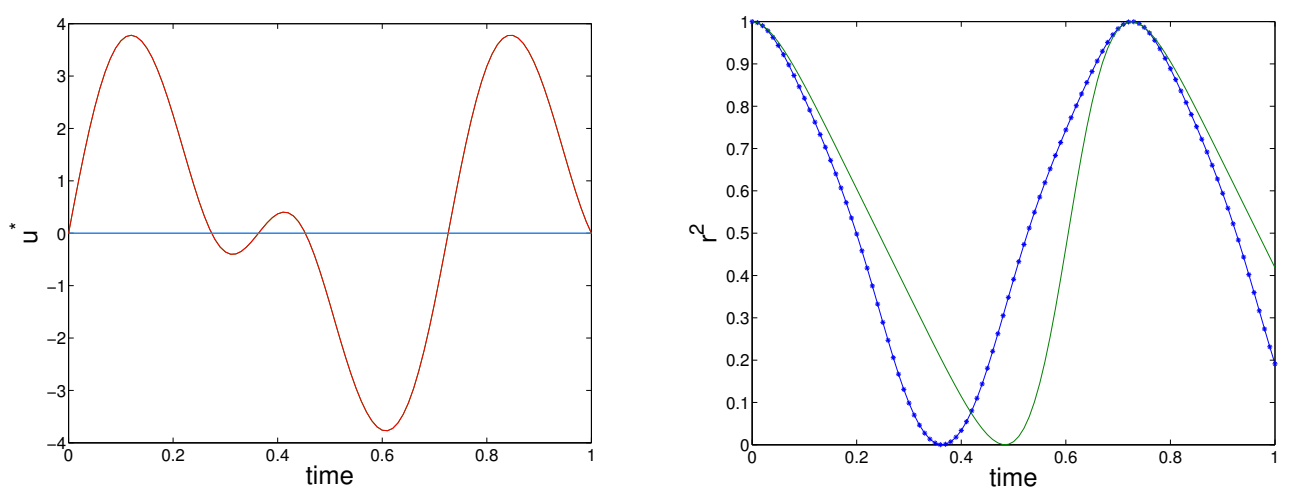

Figure 1: (a) $u_{i j}$ and (b) $r^{2}$ for $x_{0}=<0,0>, \omega=<0,10>, T=1$, and $K=5$. The synchrony is .5676 , the average coupling cost of the controlled network is 5.5873 which corresponds to $K=2.3637$. In (b) the starred line corresponds to the controlled network. The solid line corresponds to the Kuramoto network. The value of the constant adjoint variable in this simulation was 260.9995 .

Numerical simulations of Problem II, where the objective is to maximize synchrony for a bounded connectivity cost, yield similar results. Using the initial guess $u_{i j} \equiv K$ for $i \neq j$, we identify almost constant solutions of the optimality system. These solutions appear to converge to the solution $u_{i j} \equiv K$ for $i \neq j$ as $T$ grows large (see Figure 2).

The nearly constant solutions of the optimality system may be artifacts of the constant controls we use to initialize the numerical routine. Indeed, using an oscillatory coupling strength that is derived from that pictured in Figure 1 to initialize the numerical routine, we identify a beautiful oscillatory solution of the optimality system for Problem I for $K=10.1$ (Figures $3-4$ ). Figure 4 compares this oscillatory solution of the optimality system to a nearly constant solution that was found by initializing the numerical routine with a constant control. The oscillatory control is considerably more efficient than the nearly constant control. In particular, the average coupling cost of the nearly constant control is 90.3850 (which corresponds to $K=9.5071$ ), while that of the oscillatory control is 38.8299 (which corresponds to $K=6.2314$ ). 


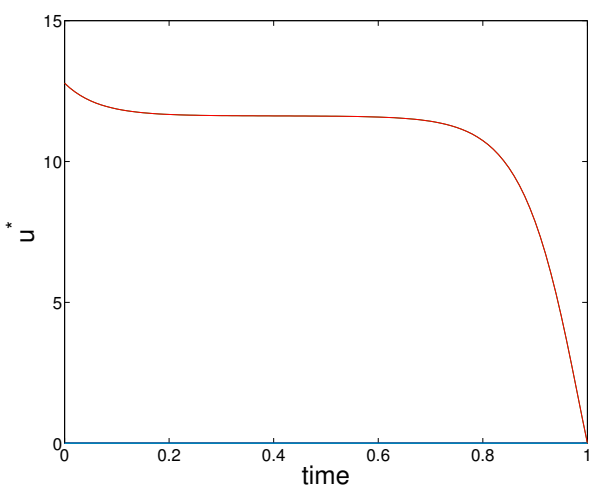

(a) $\mathrm{T}=1$

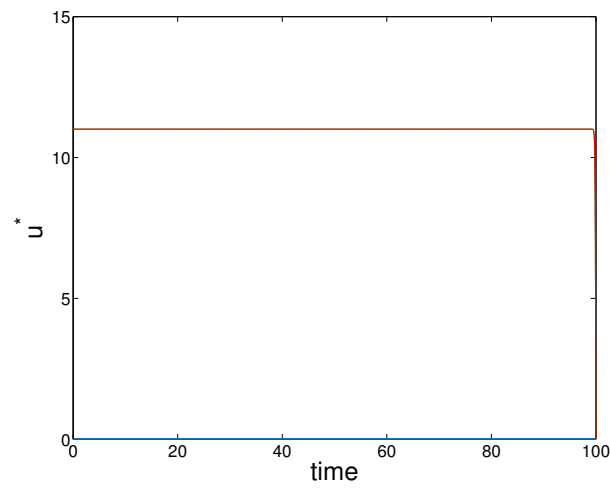

(b) $\mathrm{T}=100$

Figure 2: Comparison of $u^{*}$ for $T=1$ and $T=100$. In both simulations, $N=2, K=11$, and $\omega=<0,10\rangle$. The average synchrony of the Kuromoto network is .7080 , that of the controlled network is .7275 for $T=1$ and .7083 for $T=100$. The value of the constant adjoint variable was 742.0208 and 491.2342 in the first, respectively second, simulation.

Note that the oscillatory solution appears to improve upon the efficiency of the mean field network by allowing the order parameter to oscillate. As the order parameter undergoes large amplitude oscillations, the control preserves the total synchrony by broadening the order parameter's peaks and narrowing its valleys. This effect is even more apparent for slightly higher values of $K$ (not shown). Numerical simulations indicate that as $K$ moves away from the synchronization threshold, this oscillatory strategy becomes less and less efficient until it is surpassed by the nearly constant strategy. It seems plausible that small amplitude oscillatory solutions exist for higher values of $K$, but we are unable to identify such solutions at this time. It is also interesting to note that the solution pictured in Figure 4 exhibits negative coupling, i.e. there are times when the oscillators are repulsed instead of attracted to each other. Previously, we found that repulsion can promote synchrony in a network with constant coupling strengths [3]. Hence, it appears that repulsion can promote synchrony in a variety of contexts.

Having considered optimal time-varying coupling between a pair of oscillators, we examine the problem of coupling a group of three oscillators with $\omega=<0,10,11>$, so that the first oscillator may be considered as a sort of contrarian to the other two. For this choice of $\omega$, the network may exhibit partially synchronous states in which $x_{2}$ and $x_{3}$ are phase locked, while $x_{1}$ 


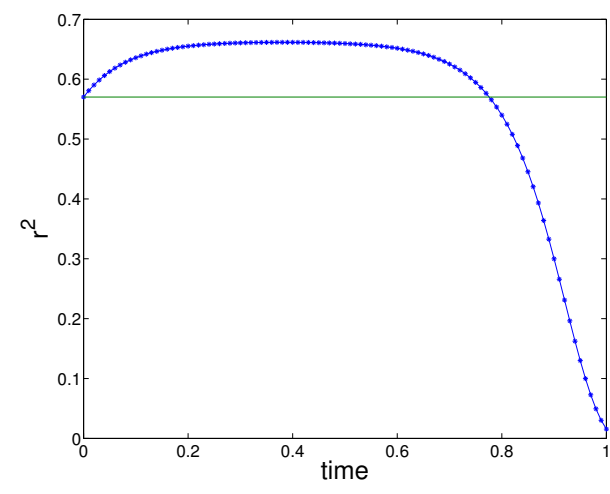

(a) a nearly constant solution

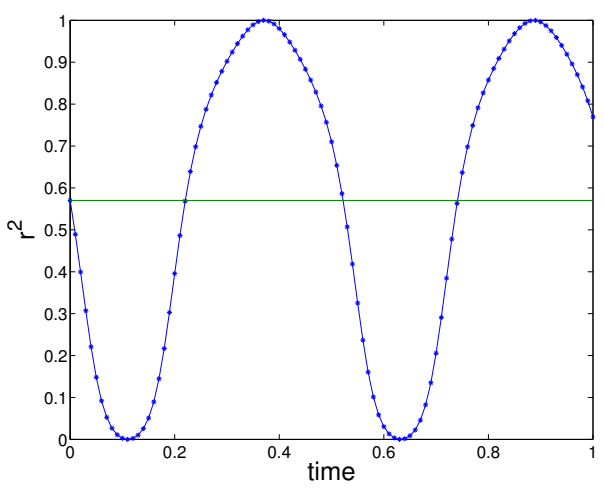

(b) an oscillatory solution

Figure 3: Multiple solutions of the optimality system: $r^{2}$ for $N=2, T=1$, and $K=10.1$. The synchrony of the network is .5699. The average coupling cost of the nearly constant control is 90.3906 , that of the oscillatory control is 38.9397 , and that of the Kuramoto network is 102.01. The starred line corresponds to the controlled network and the solid line corresponds to the Kuramoto network. The value of the constant adjoint variable is 327.5173 in simulation (a) and 15,393 in simulation (b).

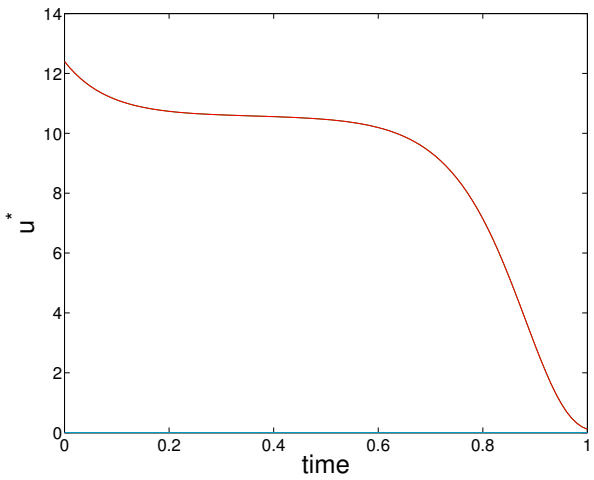

(a) a nearly constant solution

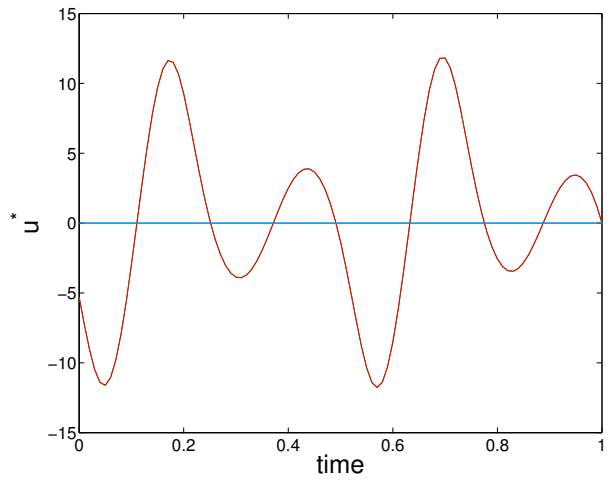

(b) an oscillatory solution

Figure 4: Multiple solutions of the optimality system: $u^{*}$ for $N=2, T=1$, and $K=10.1$. The average coupling cost of the nearly constant control is 90.3850 , that of the oscillatory control is 38.8299 , and that of the Kuramoto network is 102.01 . 
drifts. Numerical simulations indicate that in a Kuramoto network $x_{1}$ and $x_{2}$ become phase locked for $K \approx 1.5$. It seems possible that in an optimally coupled network phase locking of $x_{1}$ and $x_{2}$ may occur for lower values of $K$. Hence we perform numerical simulations in order to examine the dynamics of the population joined through an optimal time-varying network with $K=1$. The resulting oscillator phases $(\bmod 2 \pi)$ are shown in Figure 5 (b). The dynamics of $r^{2}$ for the controlled and Kuramoto network and the time-varying control are shown in are shown in Figure 6 (a) and (b), respectively. We note that the time-varying network substantially increases the synchrony of the population. In particular, the advantage of the timevarying network becomes increasingly apparent as the Kuramoto network drifts away from the initial synchronous state (see Figure 6 (a)). In addition, the time-varying network keeps $x_{1}$ and $x_{2}$ close for the majority, but not the entirety, of the simulation. Interestingly, the time-varying control appears to be antisymmetric about $t=4$ and hence exhibits considerable repulsion. In particular, the first oscillator, which has zero natural velocity, is driven to travel counterclockwise about the unit circle (see Figure 7).

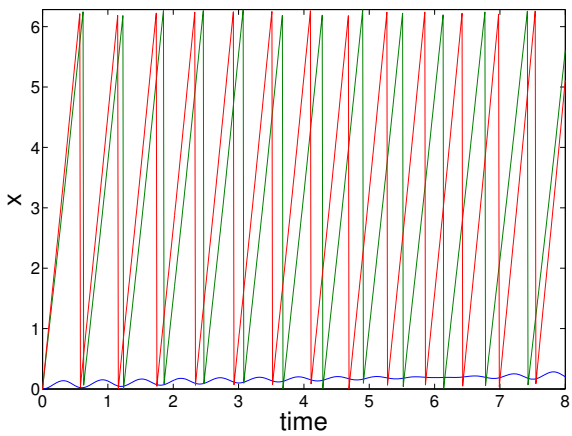

(a) Kuramoto network

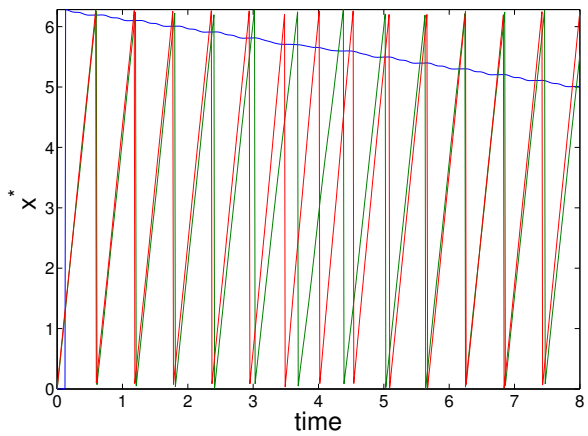

(b) time-varying network

Figure 5: a) The phases $(\bmod 2 \pi)$ and $b)$ the corresponding control of a population of three oscillators with $\omega=<0,10,11>$ with $K=1, T=8, M=100$, and $x_{0}=<0,0,0>$. Here blue corresponds to $x_{1}$, green corresponds to $x_{2}$, and red corresponds to $x_{3}$.

As with the two-oscillator network, we are unable to identify oscillatory solutions of the optimality system for this three-oscillator network for $K$ large. In particular, we find oscillatory solutions of the optimality system for $K \leq 6.5$. The trajectory of $r^{2}$ and the time-varying control for $K=6.5$ are shown in Figure 8. The average synchrony of the population joined 


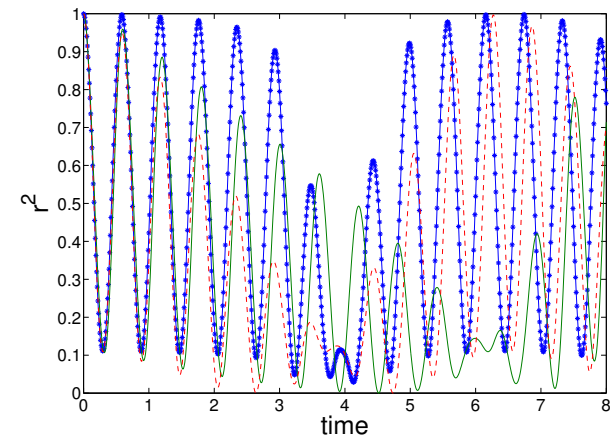

(a) $r^{2}$

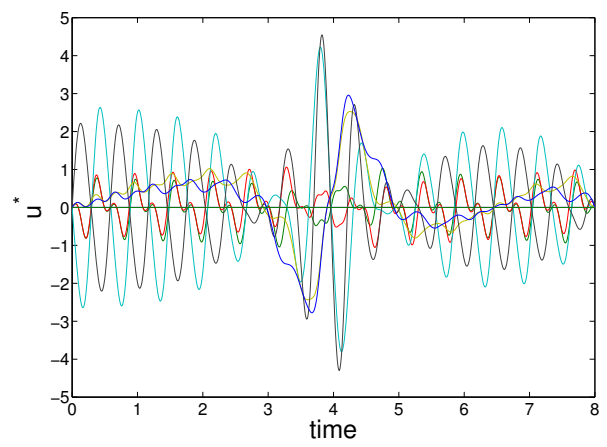

(b) control

Figure 6: a) Comparison of $r^{2}$ for a population of three oscillators joined through $\mathrm{Ku}-$ ramoto (-), constant controlled (- $)$ and time-varying controlled $\left(^{*}\right)$ networks, with $\omega=<0,10,11>, x_{0}=<0,0,0>, T=8$ and $K=1$. The average synchrony of the Kuromoto network is .3173, that of the constant controlled network is .3657 and that of the controlled time-varying network in .4896. The value of the constant adjoint variable for this time-varying control is 129.2939 .

through this time-varying network is .6272 . We note that this population of oscillators (when joined via a Kuramoto network) exhibits phase-locking at $K \approx 10.6$, at which point the asymptotic value of $r^{2}$ is approximately .6067 . Hence, these simulations support the idea that oscillatory networks are more efficient at achieving moderate levels of synchrony. These gains in efficiency are achieved by enabling the order parameter to undergo large amplitude oscillations about its average value.

Next we used this group of three oscillators to compare the efficacy of time-varying networks to promote synchrony to that of stationary heterogeneous networks. In particular, heterogeneous stationary networks were constructed in order to maximize the average synchrony of this population of three oscillators for $K=1$ and $K=6.5$ as in [3]. Simulations indicate that the time-varying network substantially outperforms the stationary network (Figures 6 (a) and 7 (a)).

Finally we consider a seven oscillator analogue of the previous network with $\omega=<0,10,10.2,10.4,10.6,10.8,11>$. Note that for this choice of $\omega$ the potential influence of the contrarian oscillator is diminished by the larger number of oscillators in the cohesive subgroup. When joined via a Kuramoto network, numerical simulations indicate that this population exhibits a partially synchronous state for $K \approx .9$. Hence we consider the problem of 


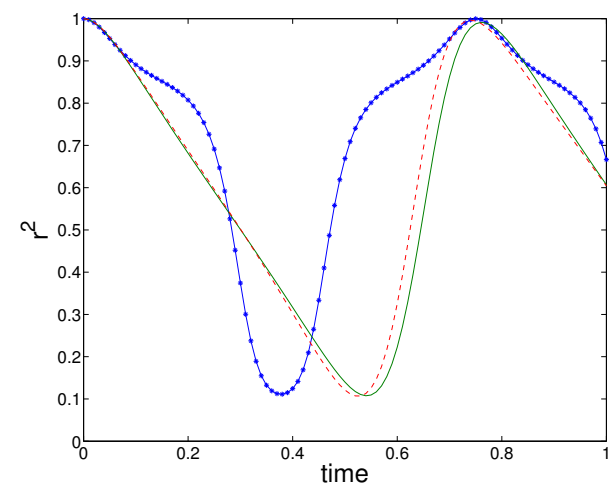

(a) $r^{2}$

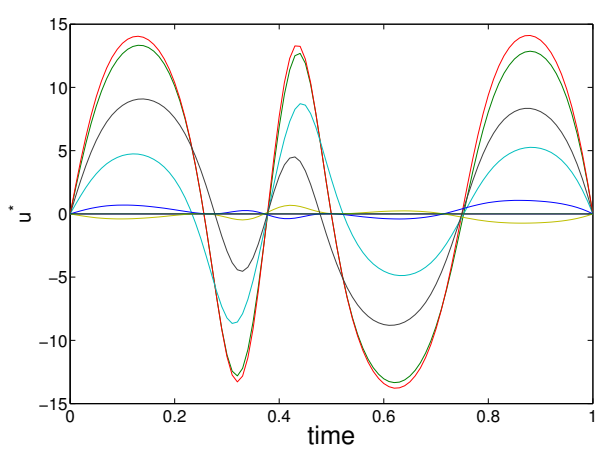

(b) $u^{*}$

Figure 7: a) Comparison of $r^{2}$ in Kuramoto (-), time-varying controlled (*), and constant controlled networks (- -). With $N=3, \omega=<0,10,11>, x_{0}=<0,0,0>, T=1$, $M=100$, and $K=6.5$. The average synchrony of the Kuromoto network is .6156 , that of the constant controlled network is .6272 and that of the controlled time-varying network is .7327 . The value of the constant adjoint variable is 14,384 for the constant control and 3,714 for the time varying control. b) The time-varying control.

constructing a time-varying network to maximize the synchrony of the population with $K=.8$. The trajectories of the resulting oscillator phases, $r^{2}$, and time-varying coupling strengths are shown in Figures $8-10$. Figures 8 and 9 show that, at least for this limited time-period, the time-varying control is able to preserve synchrony among the cohesive oscillator subgroup. Whereas, for this value of $K$, numerical simulations indicate that the order parameter of an initially synchronized population that is joined through a Kuramoto network will collapse to zero around $t=23$. As a result the average order of the population joined via the time-varying network is substantially greater (.7274) than that of the population joined through the corresponding Kuramoto network (.5600). 


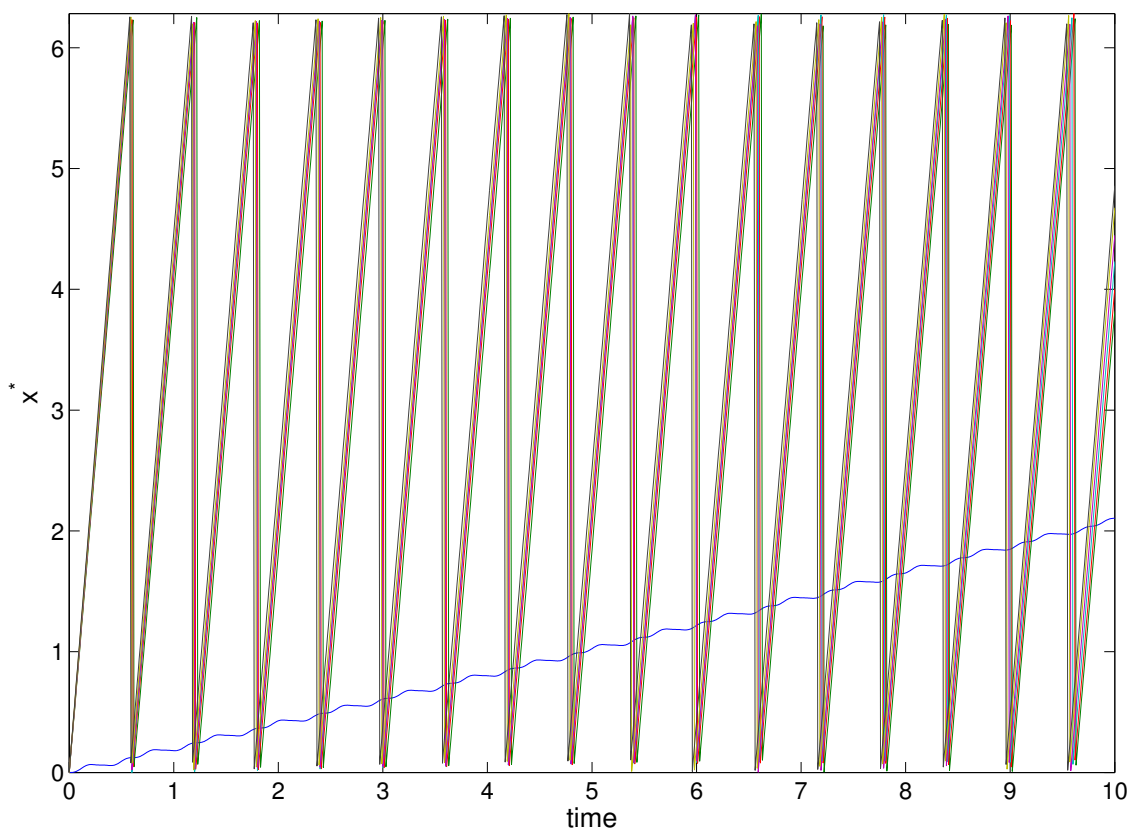

Figure 8: The phases $(\bmod 2 \pi)$ of an oscillator network with $N=7, x_{0}=<$ $0,0,0,0,0,0,0>, \omega=<0,10,10.2,10.4,10.6,10.8,11>, T=10$, and $K=.8$, joined via a time-varying controlled network. Here blue corresponds to $x_{1}^{*}$, green to $x_{2}^{*}$, red to $x_{3}^{*}$, turquoise to $x_{4}^{*}$, cyan to $x_{5}^{*}$, yellow to $x_{6}^{*}$, and gray to $x_{7}^{*}$. 


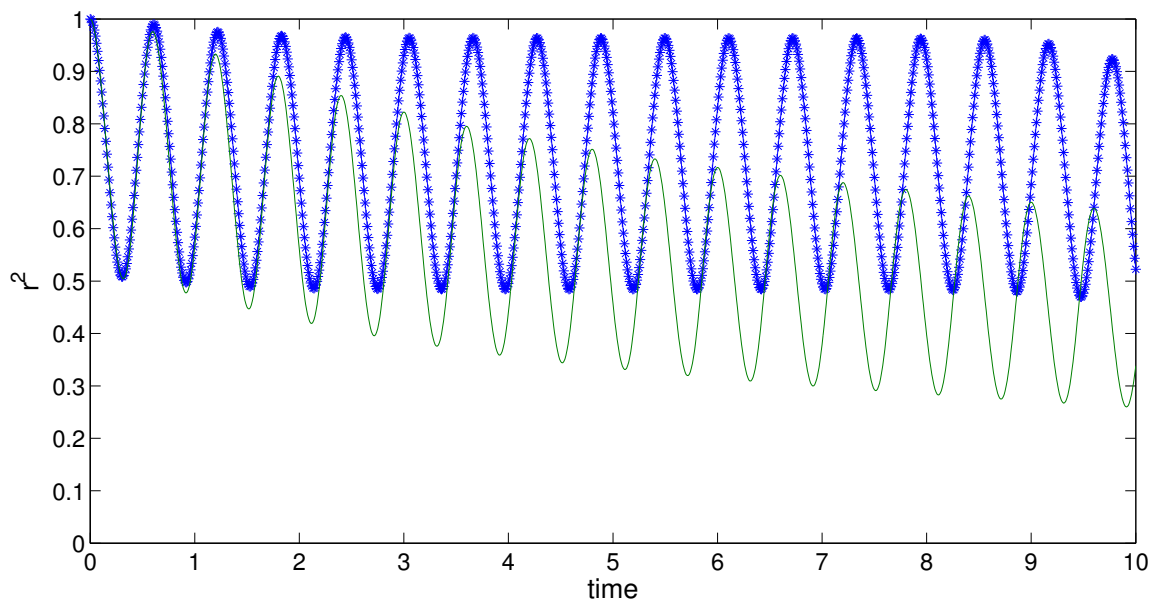

Figure 9: $r^{2}$ of an oscillator network with $\left.N=7, x_{0}=<0,0,0,0,0,0,0\right\rangle, \omega=<$ $0,10,10.2,10.4,10.6,10.8,11>, T=10$, and $K=.8$, joined via Kuramoto (-) and timevarying $(*)$ controlled networks.

\section{Conclusions}

The classical Kuramoto network is a stationary homogeneous network in which nonlinear oscillators are coupled with a constant coupling strength. Previously, we showed that heterogeneous networks can enhance synchronization [3]. In this paper, we extended our previous results to show that near the synchronization threshold dynamic networks (with non-constant coupling strengths) can be much more cost effective than static networks, be they heterogeneous or not. In particular, it appears that dynamic networks can achieve moderate levels of synchrony for reduced coupling cost. In addition, numerical simulations indicate that, as is the case for static networks, repulsion is a cost-effective means of promoting synchrony in dynamic networks. The conditions that enable the dynamic networks identified in this paper to improve upon the efficiency of the classical Kuramoto network are very unlike the conditions presented in $[4,6]$. In particular, previous results on the efficiency of dynamic networks assume that the time-scale of the network dynamics is fast compared to that of the states, so that the population behaves as if it were governed by the time-averaged network. In contrast, the dynamic networks presented here evolve on the same time-scale as the states and preserve synchrony by shortening the duration of low synchrony states 


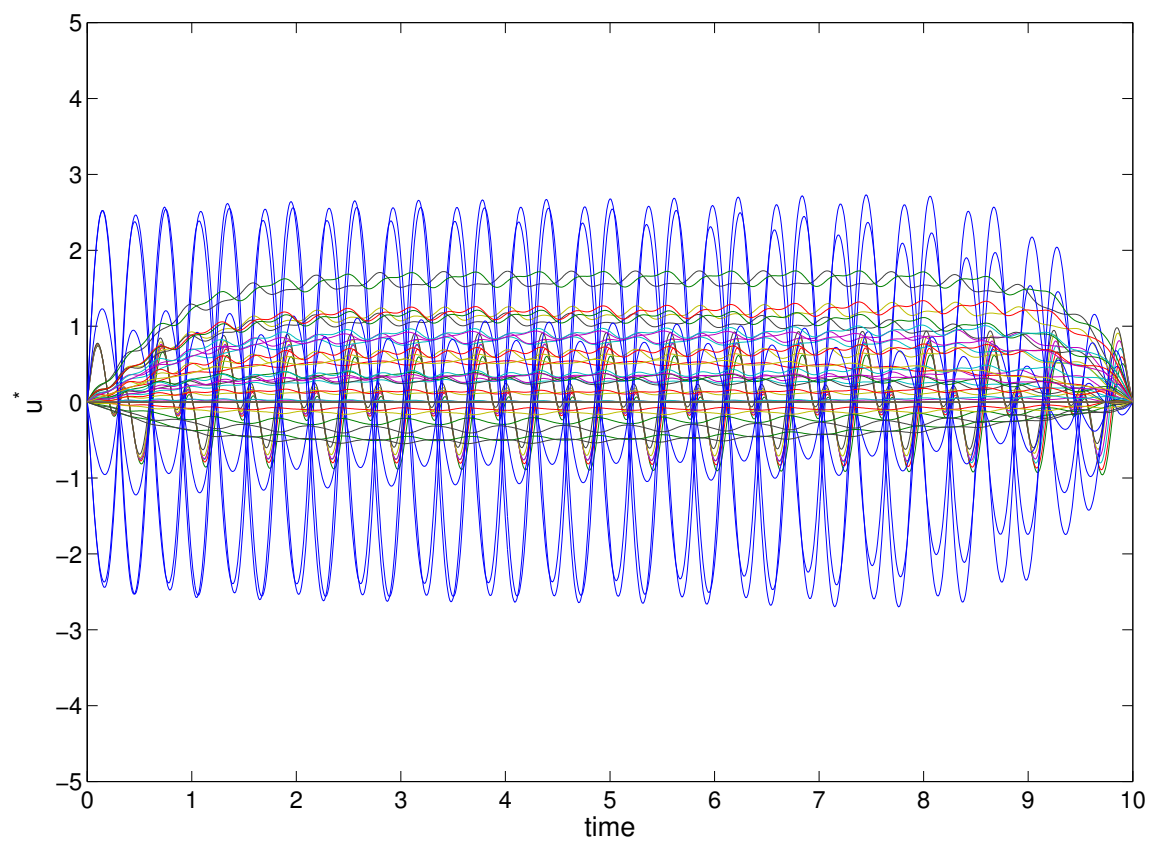

Figure 10: The dynamics of the components of $u^{*}$ for a network with $N=7, x_{0}=<$ $0,0,0,0,0,0,0>, \omega=<0,10,10.2,10.4,10.6,10.8,11>, T=10$, and $K=.8$, joined via Kuramoto (-) and time-varying $(*)$ controlled networks. The value of the constant adjoint variable for this control is 483.2597 . 
and lengthen the duration of high synchrony states. For large values of $K$, we were unable to identify any oscillatory solutions of the optimality system. However, this does not preclude the existence of such solutions. At this time numerically solving for the necessary conditions for large populations over extended intervals of time also presents a significant challenge. Future work will develop methods for overcoming these difficulties in order to provide a more complete characterization of optimal time-varying networks.

\section{Acknowledgements}

The work of Lenhart and Leander was partially supported by the National Institute for Mathematical and Biological Synthesis, sponsored by the National Science Foundation, the U.S. Department of Homeland Security, and the U.S. Department of Agriculture through NSF Award EF-0832858. Lenhart's work receives additional support from The University of Tennessee. Lenhart is also partially supported by the University of Tennessee Center for Business and Economic Research. The Oak Ridge National Laboratory is managed by UT-Battelle, LLC for the U. S. Department of Energy under contract DE-AC05-00OR22725.

[1] T. Nishikawa, A. Motter, Network synchronization landscape reveals compensatory structures, quantization, and the positive effect of negative interactions, PNAS 107 (2010) 10342-10347.

[2] T. Nishikawa, A. Motter, Maximum performance at minimum cost in network synchronization, Phys. D 224 (2006) 7789.

[3] R. Leander, S. Lenhart, V. Protopopescu, Using optimal control theory to identify network structures that foster synchrony, Physica D. 241 (2012) 574-582.

[4] I. V. Belykh, V. N. Belykh, M. Hasler, Blinking model and synchronization in small-world networks with time-varying coupling, Physica D 195 (2004) 188-206.

[5] M. Hasler, V. Belykh, I. Belykh, Dynamics of stochastically blinking systems. part ii: Asymptotic properties, SIAM Journal on Applied Dynamical Systems 12 (2) (2013) 1031-1084. arXiv:http://dx.doi.org/10.1137/120893410, doi:10.1137/120893410. URL http://dx.doi.org/10.1137/120893410 
[6] P. So, B. C. Cotton, E. Barreto, Synchronization in interacting populations of heterogeneous oscillators with time-varying coupling, Chaos 18 (2008) 037114.

[7] M. Chen, Y. Shang, Y. Zou, J. Kurths, Synchronization in the Kuramoto model: A dynamical gradient network approach, Phys. Rev. E 77 (2008) 127101.

[8] I. Belykh, M. di Bernardo, J. Kurths, M. Porfiri, Evolving dynamical networks, Physica D 267 (2014) 1-6.

[9] P. DeLellis, M. diBernardo, F. Garofalo, M. Porfiri, Evolution of complex networks via edge snapping, Circuits and Systems I: Regular Papers, IEEE Transactions on 57 (8) (2010) 2132-2143. doi:10.1109/TCSI.2009.2037393.

[10] Q. Ren, J. Zhao, Adaptive coupling and enhanced synchronization in coupled phase oscillators, Phys. Rev. E 76 (2007) 016207.

[11] S. H. Strogatz, From Kuramoto to Crawford: Exploring the onset of synchronization in populations of coupled oscillators, Physica D 143 (2000) 1-20.

[12] F. Döruler, F. Bullo, On the critical coupling for kuramoto oscillators, SIAM Journal on Applied Dynamical Systems 10 (3) (2011) 1070-1099. arXiv:http://dx.doi.org/10.1137/10081530X, doi:10.1137/10081530X.

URL http://dx.doi.org/10.1137/10081530X

[13] H. J. Sussmann, Some recent results on the maximum principle of optimal control theory, in: C. I. Byrnes, B. N. Datta, C. F. Martin, D. S. Gilliam (Eds.), Systems and Control in the Twenty-First Century, Vol. 22 of Systems \& Control: Foundations \& Applications, Birkhäuser Boston, 1997, pp. 351-372.

[14] S. Lenhart, J. Workman, Optimal Control Applied to Biological Models, Chapman and Hall/CRC Mathematical and Computational Biology Series, Chapman \& Hall/CRC, 2007.

URL http: //books.google.com/books?id=2IMoAAAAYAAJ 